\title{
Occupation Measurements Supporting Dynamic Spectrum Allocation for Cognitive Radio Design
}

\author{
V. Blaschke, H. Jaekel, T. Renk, C. Kloeck, and F. K. Jondral \\ Institut für Nachrichtentechnik \\ Universität Karlsruhe (TH), Germany \\ email: $\{$ blaschke,jaekel,renk,kloeck,fj\}@int.uni-karlsruhe.de
}

\begin{abstract}
Efficient use of radio spectrum is a necessity for future wireless systems. This can, for example, be accomplished by cognitive radios. An important point for designing future systems is to gain knowledge about the occupation of the frequency bands which may be used by the cognitive radio. This includes the general characterization of the traffic density at the frequency bands as well as a detailed analysis of the temporal frequency occupation. Thus, this paper describes measurements taken in the GSM network at three different scenarios. Furthermore, a detailed analysis of the measurement results is presented including statistical as well as spectral occupation analysis.
\end{abstract}

Index Terms-Spectrum Measurement, Spectral Utilization, Dynamic Spectrum Allocation, Cognitive Radio

\section{INTRODUCTION}

$\mathbf{T}$ HE demand for mobile services steadily increases. Often radio resources are proclaimed to be scarce resources, but this scarcity only arises due to an inefficient usage. By employing adaptive allocation and dynamic pricing mechanisms these resources can be exploited more efficiently. One possible technique to realize dynamic spectrum access may be based on spectrum pooling [1], [2].

In a spectrum pooling system the same spectrum is locally used by secondary users following proper rules to transmit wirelessly without disturbing the incumbent user. For example, spectrum pooling systems may be realized by primary systems allowing a secondary system to send in the time-frequency gaps whenever the primary users do not transmit. Thus, the secondary system needs to detect the primary system and it must be able to decide about transmitting and adapting its radio access technology to the current spectrum configuration. Assuming that the secondary system gets no signaling information from the primary system, the secondary system needs to learn about the past and act accordingly. This requirement exactly describes the goal of a cognitive radio [1],[3], which is defined as being aware of its environment and adapting its behavior according to the results of its sensing.

A lot of previous work has been conducted in the field of radio frequency measurements and dynamic spectrum access. In [4] the concepts of a dynamically sharable spectrum band and statistically multiplexed access have been introduced and their results have been evaluated by measurements. J. O. Juntunen et al. [5] performed measurements in the ISM band in the city center of Helsinki. They constituted that the allocation within a frequency band varies tremendously. The measurement data collected by M. A. McHenry in Chicago from $30 \mathrm{MHz}$ bis
$3000 \mathrm{MHz}$ were intended to clarify, among other aspects, the questions of channel occupation, how those channels are occupied (i.e., modulation scheme, data rate, etc.), and how many transmitters are present in a band and where they are located. In [6] a concept of spectral agile software defined radios has been discussed where a method for estimating underutilized spectrum bands has been presented.

The development of algorithms for the use of spectrum pooling in cognitive radios necessarily has to consider the statistical characteristics of typical wireless communication systems. Furthermore, the algorithms (e.g., for reducing interference and finding spectrum gaps) have to be tested for several specific situations regarding the usage of a primary system. Therefore, the major incentive of this paper is to present measurements of a typical primary system, thereupon describing the statistical characteristics and discussing potential consequences for the design of cognitive radios.

The remainder of the paper is organized as follows: In section II the measurement system is described by specifying the equipment used and the parameters chosen. The scenarios in which the measurements have been performed are characterized in section III. Measurements provide information about the time-frequency occupation of the frequency range under consideration. This time-frequency occupation serves as starting point for the statistical analysis conducted in section IV. Possible impacts of the results on the design of cognitive radios are discussed in section V. Finally, the paper is summarized.

\section{Measurement System}

The measurement equipment mainly consists of an antenna, a spectrum analyzer and a computer (see figure 1). The intention of the measurement is to observe the spectrum in proper scenarios. Based on the multi-path characteristics of the several detected parties, the direction of arrival is not known, so that an omni-directional antenna has been used. The antenna is a double conical antenna. Its horizontal direction radio pattern is relatively omni-directional with $4.1 \mathrm{~dB}$ gain measured in the proper frequency range. In contrast to this, the vertical direction radio pattern is clubbed. Thus, the whole pattern is comparable to the one of a $\lambda / 2$ dipole. The antenna input is adapted to $Z=50 \Omega$, leading to an apex angle between the two cones of $\alpha=46.4^{\circ}$ according to [7]:

$$
Z=120 \cdot \ln \left(\cot \left(\frac{180^{\circ}-\alpha}{4}\right)\right) \text {. }
$$




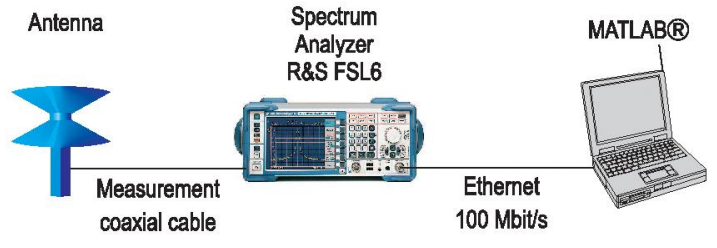

Fig. 1. Measurement equipment configuration.

Thus, the antenna and the coaxial cable are matched and no reflexion occurs. The coaxial cable transfers the signal to the spectrum analyzer FSL6 from Rohde \& Schwarz (R\&S). To measure the downlink of a wireless communication system with channels of $200 \mathrm{kHz}$ bandwidth, a resolution of $70 \mathrm{kHz}$ has been chosen. The resolution bandwidth $B_{I F}$ has been set to $100 \mathrm{kHz}$ and the video bandwidth to $10 \mathrm{kHz}$. The minimal sweep time can be calculated according to [8]:

$$
T_{\text {min }}=k \cdot \frac{\Delta f}{B_{I F}^{2}}=17.5 \mathrm{~ms},
$$

where $k$ has been set to 2.5 because the spectrum analyzer internally uses a Gaussian-sweep filter for such a wide frequency range and not the FFT. Therefore, the sweep time has been set to $115 \mathrm{~ms}$. Finally, a program written in $\mathrm{C}++$ has read out the power level measured in $\mathrm{dBm}$ every $115 \mathrm{~ms}$ and stored the data in several short files, to reduce access time. Afterwards, the data has been processed in Matlab ${ }^{\mathbb{R}}$. These results will be presented in the following sections.

\section{SCENARIO DESCRIPTIONS}

In the measurement campaign three different scenarios have been addressed: scenario 1 is a big event with several thousands of people in a small area in a big city, section 2 devotes to the neighborhood of such a big event and scenario 3 serves as reference measurement for a normal load in a big city. In the following, the three scenarios will be described.

\section{A. Scenario 1}

In current cellular radio system, the cells and the cell structure are designed to cope the normal traffic demand. The first question arises, how the spectrum of cellular radio system looks like at a big event at which several thousands of people attend. Intuitively, a highly used spectrum has been expected, which was a correct supposition by further consideration of the measurement results. The thought behind was to clarify if spectrum pooling is always possible, or if there could be situations in which spectrum pooling is not suitable, because all parties would need more spectrum to serve their customers. This is a drawback of the absence of application diversity for frequency-adjacent systems of the same radio access technology. Furthermore, the spectrum occupation has been expected to increase just before the big event started and to decrease when the crowd left. This can be seen in the measurements results. Finally, any big event has breaks, thus, are there any correlations of the load and these breaks? In turn, this phenomenon can be observed in the measurement analysis results. The measurement was placed in a big city.
The measurement equipment has been placed high above this hot spot, in order to possess line of sight to the hot spot and to detect as much base stations (BS) serving the hot spot as possible.

\section{B. Scenario 2}

Considering scenario 1, a question may arise about load distribution of such an hot spot in space. Therefore, this scenario 2 is placed next to such an event of comparable size in order to measure the load in the neighborhood cells. One suggestion can be that before and after the event the load might be increased because the crowd arrives and leaves, respectively. This can be seen as a moving hot spot. Unfortunately, the measurement results do not show this characteristic. This shows, that if there was a moving hot spot, it came from another direction, or the hot spot was created locally. The measurement place was in a big city. The measurement equipment has been placed above the streets to increase as much as possible the probability to detect the adjacent BSs.

\section{Scenario 3}

This scenario serves as reference scenario, to compare the results of both aforementioned scenarios with a normal day and normal load. The measurement location and execution has the same characteristics as in the other both scenario, but without a big event.

\section{Measurement Results}

\section{A. Detection Threshold}

Measurements are a proper means to evaluate occupation and other statistical characteristics of wireless communication systems. However, an important issue that occurs with measurements is a suitable determination of a detection threshold if predications on occupied and non-occupied channels at certain times should be made. Therefore, two possibilities exist, namely an empirical and an analytical method.

The empirical method merely uses the obtained measurement results and makes the following assumption: Since there are a lot of superposing noise sources in a communication environment, one may state due to the central limit theorem that the sum of all noise sources possesses a Gaussian power level distribution. Thereby, the only assumption is that all sources are independent. In a next step, first and second order moments must be calculated, which means average value $\mathcal{E}\{\cdot\}$ and variance $\mathcal{V}\{\cdot\}$. For this purpose, a channel beyond the normally utilized frequency range that is not allocated by other users is measured (in spite of knowing that this specific channel is a "noise" channel the calculation of mean value and variance are necessary because of the statistical characteristics of the channel). In a last step, the detection threshold, $P_{\text {th }}$, can be defined as

$$
P_{\mathrm{th}} \stackrel{!}{=} \mathcal{E}\left\{P_{n}\right\}+\kappa \cdot \mathcal{D}\left\{P_{n}\right\},
$$

where $\mathcal{E}\left\{P_{n}\right\}$ denotes the mean value of the power the measured channel, $\mathcal{D}\left\{P_{n}\right\}$ is the corresponding standard deviation, 
and $\kappa$ is a factor to determine the deviation's range. In our case we obtained the values

$$
\begin{aligned}
& \mathcal{E}\left\{P_{n}\right\}=-95.4 \mathrm{dBm} \\
& \mathcal{D}\left\{P_{n}\right\} \approx 1.53 \mathrm{dBm}
\end{aligned}
$$

and set $\kappa=3$. From this it follows that more than $99.7 \%$ of all noise values are reliably detected as noise. Finally, we chose a detection threshold of

$$
P_{\mathrm{th}}=-90 \mathrm{dBm}
$$

Obviously, analyzer settings influence the obtained measurement data sets. However, if we use the empirical method, this will only be considered indirectly. One possibility to include the analyzer settings (resolution bandwidth, video bandwidth, sweep time, etc.) and internal influences of the analyzer (mixer, amplifier, etc.) directly in the calculation of the detection threshold is the analytical method. The average noise power level can be calculated as

$$
\begin{aligned}
\mathcal{E}\left\{P_{n}\right\}= & -174 \mathrm{dBm}(\mathrm{Hz})+10 \log _{10}\left(\frac{B_{I F}}{\mathrm{~Hz}}\right) \mathrm{dB} \\
& +\mathrm{NF}-2.5 \mathrm{~dB},
\end{aligned}
$$

where $-174 \mathrm{dBm}(\mathrm{Hz})=10 \log _{10}\left(k_{B} T / \mathrm{Hz}\right)$ describes the Johnson noise, $k_{B}$ is Boltzmann's constant $\left(1.38 \cdot 10^{-23} \mathrm{~J} / \mathrm{K}\right)$, $T$ is the temperature (set to $293 \mathrm{~K}$ ), $B_{I F}$ is the resolution bandwidth of the spectrum analyzer (set to $100 \mathrm{kHz}$ ), NF is the noise figure of the spectrum analyzer, which is denoted as $31 \mathrm{~dB}$, and $2.5 \mathrm{~dB}$ are due to the selected detector and logarithmic scaling [9]. With these values, we obtain

$$
\mathcal{E}\left\{P_{n}\right\}=-95.5 \mathrm{dBm}
$$

Admittedly, one only obtains the mean value of noise power and no exact detection threshold. This threshold must be set by introducing a certain hysteresis. Nevertheless, the analytical method provides an opportunity for a quick estimation without the need to built up the measurement equipment.

The detection threshold can be used to create allocation vectors with respect to specific frequencies (or allocation matrices with respect to several frequencies). Figure 2 depicts a spectrogram of the measurements performed in the close vicinity to a mega-event. Normally, a spectrogram is a threedimensional plot that displays the power of a signal versus time and frequency. The vertical axis represents time, the horizontal axis represents frequency (these assignments may change in different applications) and the third axis represents power level. Often a spectrogram is reduced to only two dimensions. In this case, higher portions of power are indicated with more intense gray values. A spectrogram can also be called a spectral waterfall or sonogram.

To calculate the spectrogram the short time Fourier transform is used. In the time domain, a digitally sampled signal is divided into several frames which usually overlap. Every frame is then Fourier transformed to calculate the magnitude of the frequency spectrum for each frame. Each frame represents one horizontal line of the spectrogram. A combination of all

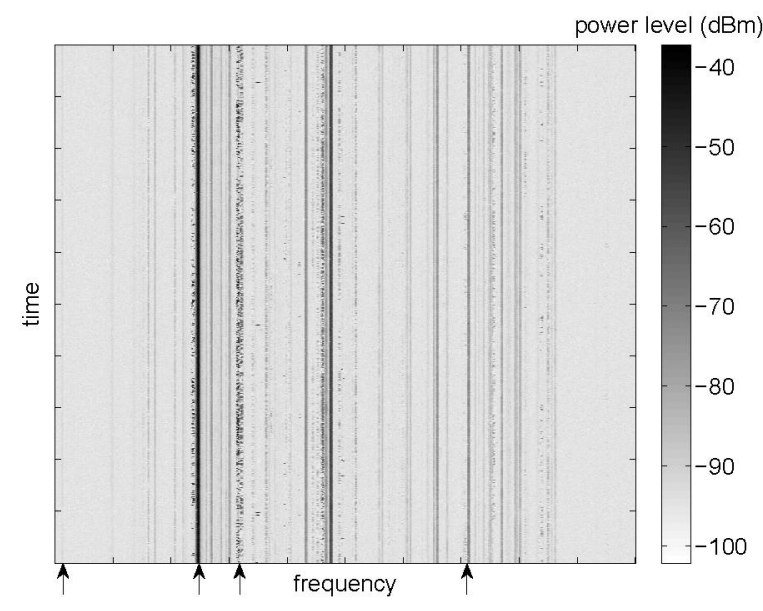

Fig. 2. Spectrogram of measurements in scenario 2 .

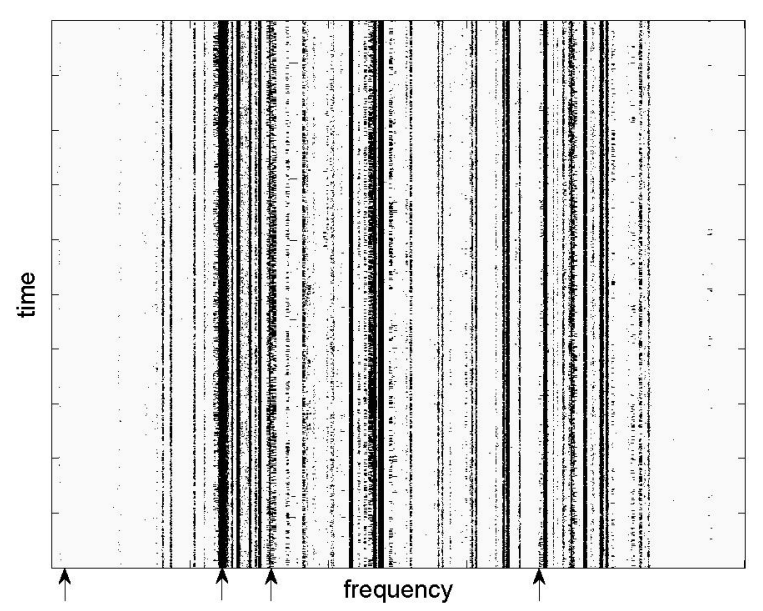

Fig. 3. Binary spectrogram of measurements in scenario 2.

Fourier transformed frames then forms the spectrogram. The short time Fourier transform, $X(f, \tau)$, is defined as follows:

$$
X(f, \tau)=\int_{-\infty}^{\infty} x(t) \cdot w(t-\tau) \cdot e^{-j 2 \pi f t} d t
$$

where $w(t)$ is the window function, and $x(t)$ is the signal to be transformed. In the measurements a Gaussian window function $w(t)$ is applied. It is a complex function that represents phase and magnitude of the signal over time and frequency. Eventually, the squared magnitude of the short time Fourier transform yields the spectrogram of the function:

$$
\mathcal{S}\{x(t)\}=|X(f, \tau)|^{2}
$$

The allocation matrices represent a binary spectrogram, $\left.O\{x(t)\}\right|_{f=f_{m}}, m=1,2, \ldots, M$, where 1 stands for allocated and 0 stands for not allocated. An allocated frequency at a specific time interval is then displayed as a black dot (see figure 3).

$$
\left.O\{x(t)\}\right|_{f=f_{m}}= \begin{cases}1 & \text { for }\left.\mathcal{S}\{x(t)\}\right|_{f=f_{m}}>P_{\mathrm{th}} \\ 0 & \text { for }\left.\mathcal{S}\{x(t)\}\right|_{f=f_{m}} \leq P_{\mathrm{th}}\end{cases}
$$


For the sake of simplicity, the expression $O_{t, f_{m}}$ will be used for the description of the binary spectrogram in the remainder of the paper.

\section{B. Complementary Cumulative Distribution Function}

The instantaneous envelope power $P$ of a complex signal can be calculated with the equation

$$
P=P_{I}^{2}+P_{Q}^{2}
$$

where $P_{I}$ and $P_{Q}$ represent the power of the in-phase and quadrature phase of the waveform, respectively. However, signals in communication environments possess a random behavior. That is why a proper statistical description of the signal is necessary to be able to extract useful information. This is done by a complementary cumulative distribution function (CCDF). A CCDF curve expresses how much time a signal spends at or even above a specific power level. In order to describe the development of a CCDF curve mathematically assume a signal $x(t)$ that has the probability density function $p_{X}(x)$. To obtain the cumulative density function, $F_{X}(x)$, the probability density function is integrated:

$$
F_{X}(x)=\int_{-\infty}^{x} p_{X}(\xi) d \xi .
$$

In a last step, the complementary is calculated by:

$$
\operatorname{CCDF}(x)=1-F_{X}(x) .
$$

Figures $4-6$ show CCDF curves of a communication system at a mega-event, and in an urban cell of a medium size city. Three types of channels can be identified in all three cases, namely noise channels (power levels minor $-90 \mathrm{dBm}$ ), channels with statistical occupation (traffic channels), and beacon channels that transmit a signal all the time, thus having a quite high power level for almost the whole observed time period. For example, consider in figure 4 the curve with the circle markers. It can easily be seen that signal power in this observation period is lower than $-90 \mathrm{dBm}$ which indicates that this channel is just a noise channel without any occupation. The mean value of this curve is $-95.2 \mathrm{dBm}$ and matches the calculated one of the analytical method for the determination of the detection threshold (see Section IV-A). The curve with the square markers surely is a traffic channel. For instance, the power level on this channel is above $-65 \mathrm{dBm}$ for $30 \%$ of the observation period. The curve with the triangle markers, denoted as Traffic Ch. 2 in figure 4, is also a traffic channel. The beacon channel is the one with the star markers. This marking is also used in figure 5 and 6.

\section{Power Histogram}

Besides the CCDF curves, the histogram of the measured power can also be used for characterization. In order to analyze each scenario we calculated the histogram of all measured frequencies for the complete monitoring period and for each traffic channel. The results are depicted in figures $7-9$. The overall histogram characterizes the general traffic density within the monitored environment. As it can be seen in figure 7 the general level of the measured power at the mega-event is significantly higher than the power level at scenario 2

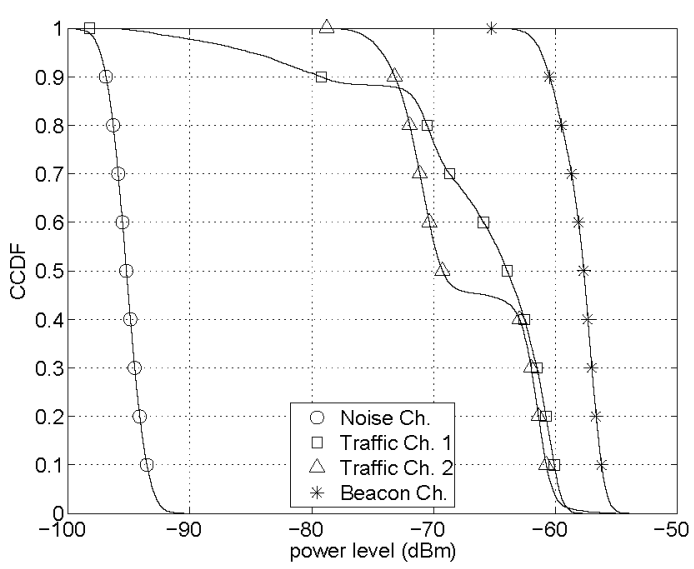

Fig. 4. CCDF curves of measurements in scenario 1.

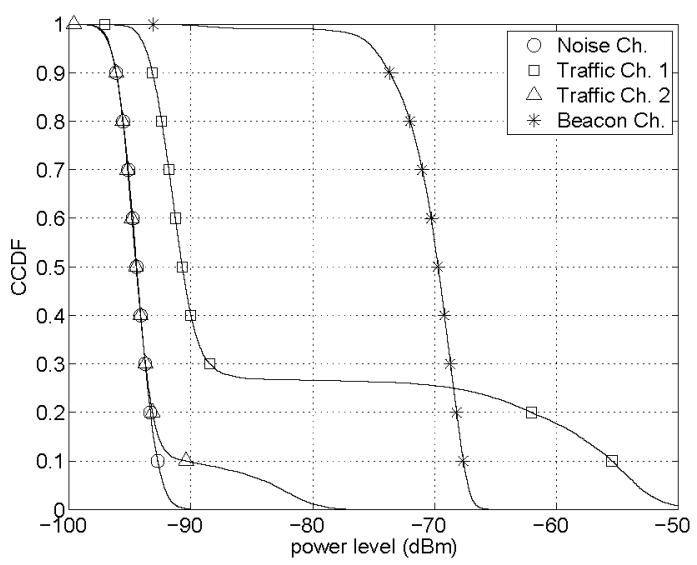

Fig. 5. CCDF curves of measurements in scenario 2.

(figure 8) or scenario 3 (figure 9). This can also be observed comparing the mean values of each histogram. The mean power at the mega-event is $-76.9 \mathrm{dBm}$. It can be noticed, that the histogram of scenario 1 has a local maximum at an averaged power of $P_{a v}=-65 \mathrm{dBm}$ due to the high number of users. Furthermore, the high standard deviation of $13.71 \mathrm{dBm}$ approves the assumption of a very busy radio environment. In the close vicinity to the mega-event the overall spectrum utilization decreases significantly which can be noticed at the lower mean value of $-92.2 \mathrm{dBm}$ (cf. figure 8 ). But the observed standard deviation of $6.91 \mathrm{dBm}$ characterizes a medium traffic density. This means, that there is a low number of traffic channels within the monitored frequency range but the traffic density on these channels is still at a medium level. In contrast to scenario 1 the mean value of scenario 3 is only $-92.8 \mathrm{dBm}$ and the standard deviation is $3.76 \mathrm{dBm}$ which characterizes a constant spectral occupation. This low and constant utilization of this environment offers the advantage of a well-predictable channel occupation which simplifies the allocation mechanisms for an overlay system.

In figure 10 the histograms of the traffic channels at scenario 1 are shown. The traffic channel 1 is depicted in black 


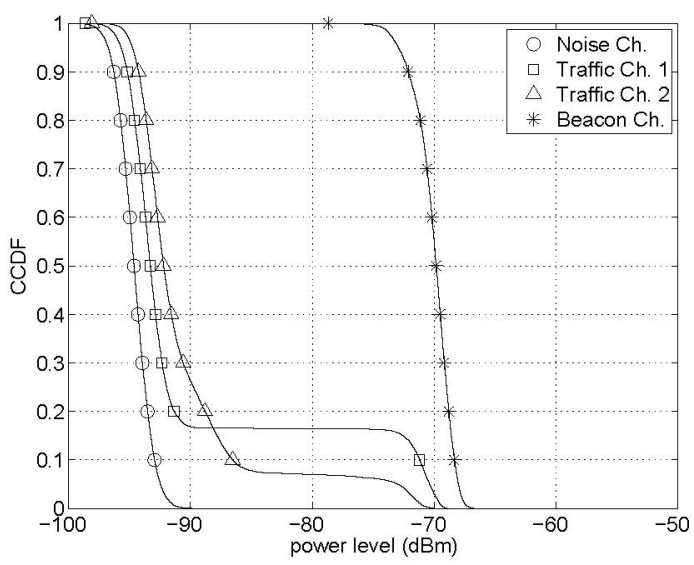

Fig. 6. CCDF curves of measurements in scenario 3.

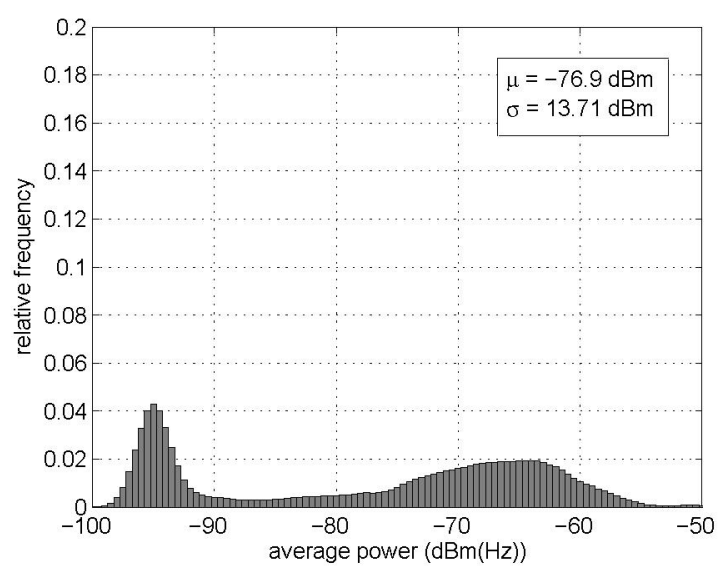

Fig. 7. Histogram of the power measured in scenario 1.

bars, traffic channel 2 in white bars with black edges. Also each traffic channel of the mega-event shown in figure 10 is characterized by a high channel occupation. The mean value of traffic channel 1 is $-83.3 \mathrm{dBm}$ and traffic channel 2 has a mean value of $-69.4 \mathrm{dBm}$, which is significantly higher than in both other described environments. The peak of the averaged power between $-100 \mathrm{dBm}$ and $-90 \mathrm{dBm}$ characterizes the noise floor of the unallocated channels. The higher the fraction of noise of the histogram classes is, the lower the spectral utilization within the monitored spectrum will be.

Furthermore, comparing the traffic channel histograms of the scenarios with each other it can be figured out that scenario 3 can be assumed as a very static environment. On the other hand, due to the high number of used chart bars in figure 7 , the spectral environment at the mega-event can be assumed as very dynamic, which also approves the expectations from the beginning. With the calculation of the averaged power histograms only information about the general characteristics but not the the temporal variation within each scenarios can be figured out.

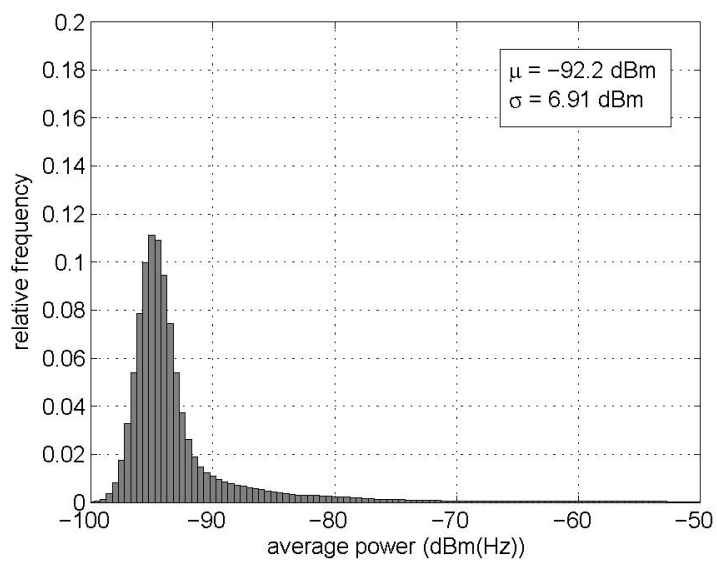

Fig. 8. Histogram of the power measured in scenario 2.

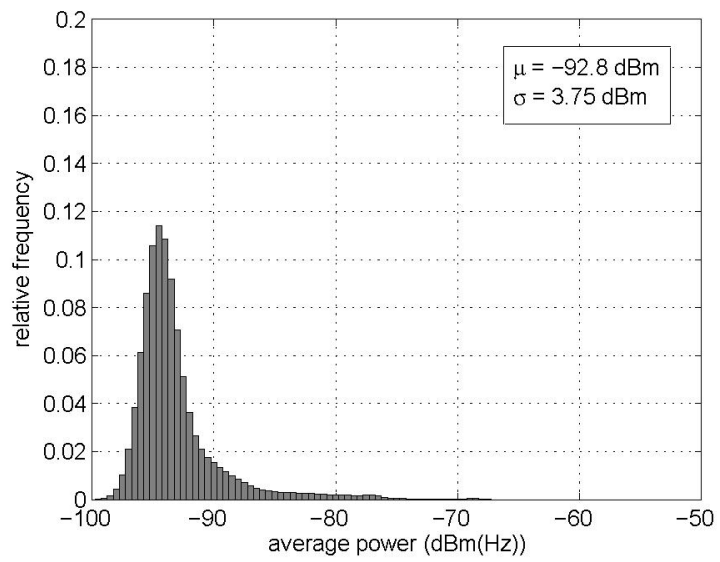

Fig. 9. Histogram of the power measured in scenario 3 .

\section{Channel Occupation}

In order to evaluate the availability of a spectral range for secondary usage the general spectral utilization over the time has to be analyzed. In [10] und [11] occupation measurements between $30 \mathrm{MHz}$ and $6 \mathrm{GHz}$ are described. The resulting averaged spectrum utilization is only about 15 percent. The averaged spectral occupation within the frequency range observed in this article can be calculated by

$$
\bar{O}=\frac{1}{N \cdot M} \sum_{n=1}^{N} \sum_{m=1}^{M} O_{t_{n}, f_{m}},
$$

where $O_{t_{n}, f_{m}}$ is the binary spectrogram at measurement time $t_{n}$ and frequency $f_{m}$ (cf. eq. (9)), $N$ is the number of all measurement points and $M$ is the number of all observed frequencies. The averaged utilization in all of our measurements averaged over all frequencies and all the observation time is about $\bar{O}=0.43$ assuming a detection threshold of $P_{\mathrm{th}}=-90 \mathrm{dBm}$. Having a look to the averaged utilization within each scenario the result significantly varies. The averaged spectral utilization at the mega-event is 0.91 , at the close vicinity 0.20 and in the normal urban environment 0.19 . Due to the high variation of the spectral utilization 


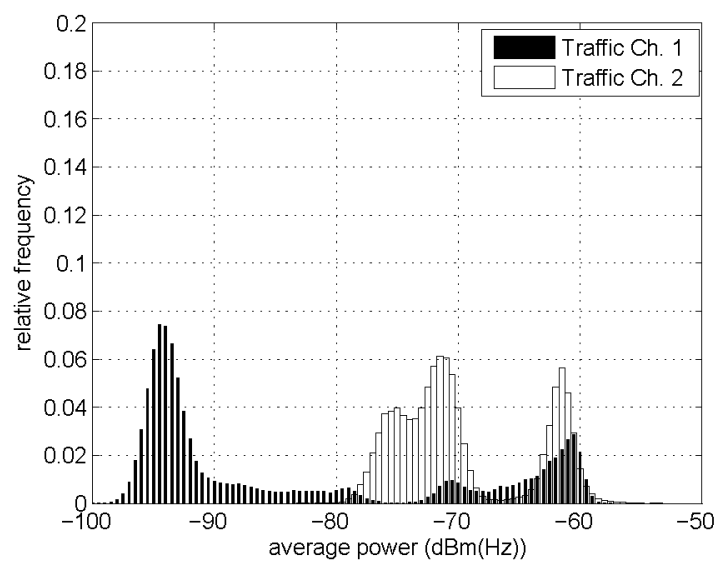

Fig. 10. Histogram of the Traffic Channel 1 and 2 in scenario 1.

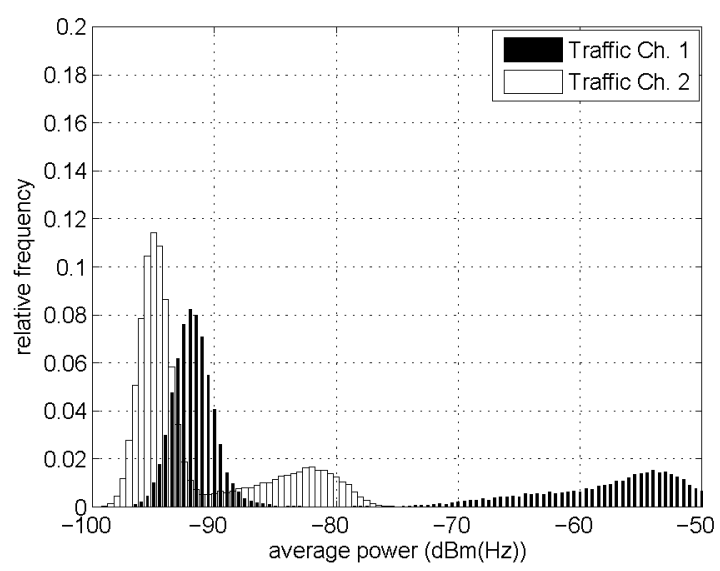

Fig. 11. Histogram of the Traffic Channel 1 and 2 in scenario 2.

within the GSM spectrum the consideration of the averaged allocation over frequency and time is not a sufficient criterion for the evaluation of the utilization which is available in fact. Therefore, we will have a closer look to the spectral utilization within each scenario. The current workload within the monitored frequency range can be figured out by using an observation window over the time. This windowed averaged occupation can be described as

$$
\overline{O_{t_{n}}}=\frac{1}{M \cdot N_{w}} \sum_{n=l}^{l+N_{w}-1} \sum_{f=f_{1}}^{f_{M}} O_{t_{n}, f_{m}},
$$

In (14) the index $l$ denotes the current sample of time, the window length is described by $N_{w}$ and is set to $N_{w}=120 \mathrm{~s}$. The resulting windowed average spectral allocation over the monitoring period is depicted in figure 13. In figure 13 the typical workload of a mega-event can be noticed. The overall utilization of this scenario is between $\bar{O}=0.91$ and $\bar{O}=0.96$. This characterizes a fully loaded mobile system, which cannot handle much more traffic. The remaining spectral capacity of several percent is caused by the general system design of GSM. As it can be seen in figure 2 due to system specific and regulatory reasons not all available GSM frequency channels

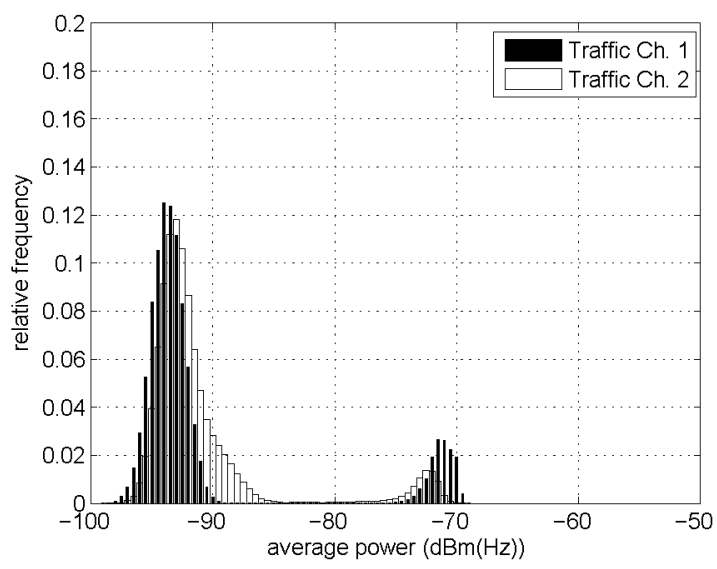

Fig. 12. Histogram of the Traffic Channel 1 and 2 in scenario 3 .

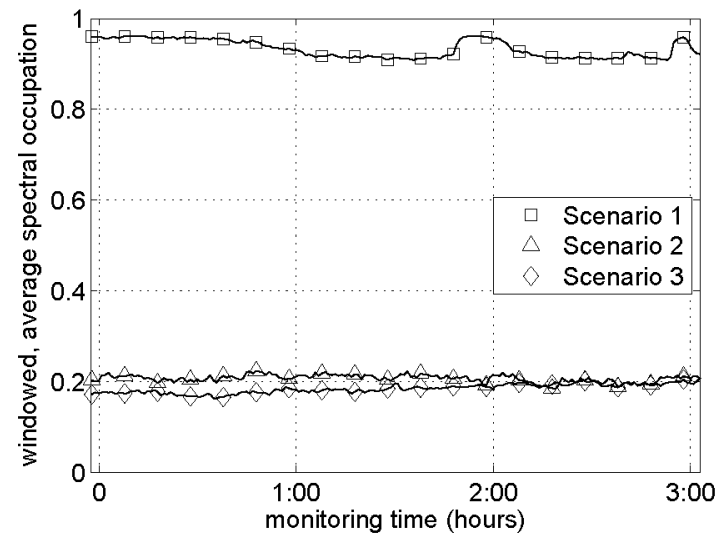

Fig. 13. Windowed average spectral utilization in the three scenarios over the monitoring period.

can be allocated. Additionally, in scenario 1 it can be shown that frequency channels which are not allocated by the system will also be detected as occupied due to the high noise floor level. This results from the high number of users within the area. If the detection threshold would be adapted depending on the current scenario and the actually used traffic channels the number of available channels for an overlay system could be increased. The resulting spectral utilization assuming a detection threshold of $P_{\mathrm{th}}=-75 \mathrm{dBm}$ is $\bar{O}=0.76$. Of course, due to the high noise floor and the worse signal-to-noise-andinterference-ratio (SNIR) the additional transmission capacity will be available only in a short range to the base station. But in such a scenario the majority of the users are located inside a small area.

In figure 14 the average channel occupation for traffic channels 1 and 2 is depicted. As discussed before, due to the high spectral power density within this frequency range and the fixed detection threshold $P_{\mathrm{th}}=-90 \mathrm{dBm}$ traffic channel 2 would be detected as allocated over the complete monitoring time. Having a look to the curve representing the channel occupation with a detection threshold $P_{\mathrm{th}}=-75 \mathrm{dBm}$ the real channel occupation can be noticed. In figure 14 also a high 


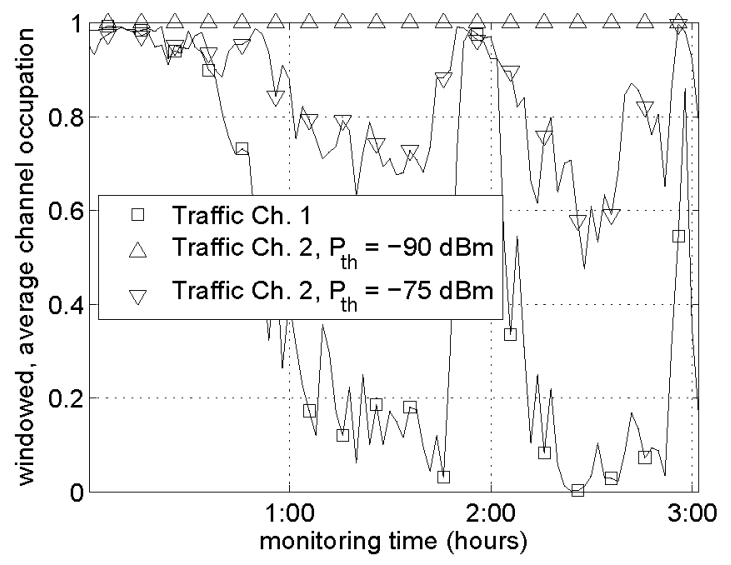

Fig. 14. Windowed, average channel occupation of traffic channel 1 and 2 (with detection threshold $P_{\mathrm{th}}=-90 \mathrm{dBm}, P_{\mathrm{th}}=-75 \mathrm{dBm}$ ) in scenario 1 .

variation of the channel occupation during the mega-event can be noticed. Depending on the characteristic of the event typical allocation patterns can be expected. The peak in the middle and at the right end of the square marked curve in figure 7 depict the breaks of the event.

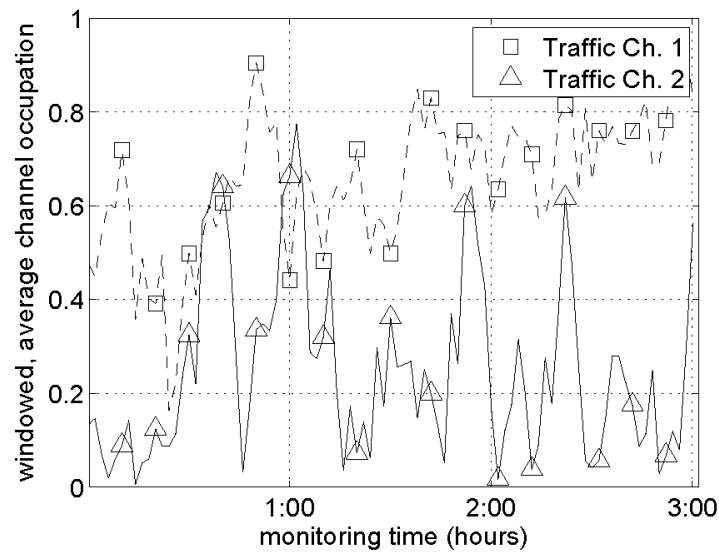

Fig. 15. Windowed, average channel occupation of traffic channel 1 and 2 in scenario 2

In figure 13 also the windowed average spectral occupation of scenario 2 and 3 is shown. It can be figured out that both curves have a similar characteristic. The spectral utilization within both scenarios is only about 20 percent. This leads to the conclusion that even in the close vicinity of a mega-event the allocation characteristic is nearly the same as in a normal urban environment. The expected moving hot spots before and after the event could not be detected, which could be caused by the measurement location. However, the low occupation is additionally motivated by the local characteristic of the megaevent scenario. Keeping this under-utilization in the close vicinity and the maximum system occupation in the center of a mega-event in mind a dynamic spectrum allocation seems to be appropriate and even necessary. Furthermore, the measured average utilization of only 20 percent offers a high potential for a secondary usage of this frequency range. The system proposed in [2] offers the possibility to use the unallocated transmission capacities of an established GSM system.

Having a look to the averaged utilization within the monitoring window at a single traffic channel the approach for secondary usage of the frequencies is motivated additionally. The allocation of traffic channel 1 and 2 at a normal usage situation in a medium sized city is shown in figure 16 . So, the majority of the spectral transmission capacity will not be used within this environment. This offers the opportunity for a local secondary usage of the remaining unallocated transmission capacity. As it can be seen in figure 15 the occupation of single traffic channels has a high variation. Although the overall spectral utilization is only about $\bar{O}=0.2$ the single channel occupation can be up to $\overline{O_{f_{m}}}=0.9$ within a period of $N_{w}=120 \mathrm{~s}$.

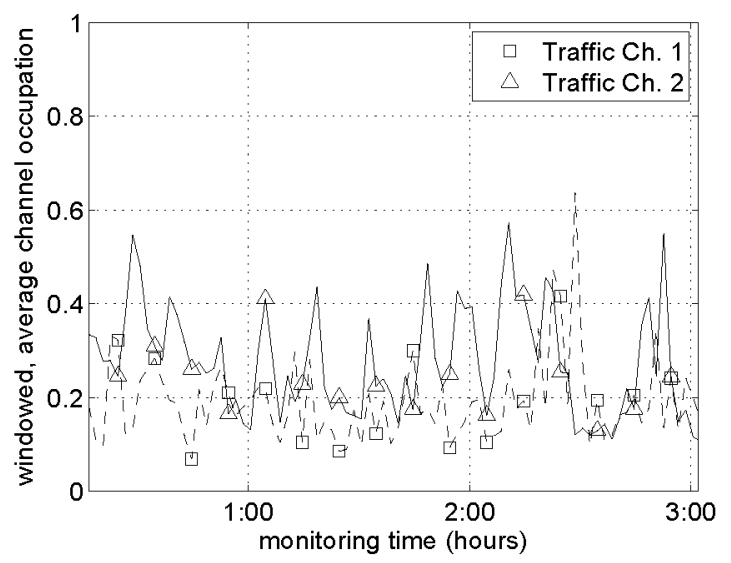

Fig. 16. Windowed, average channel occupation of traffic channel 1 and 2 in scenario 3 .

\section{Outlook to Cognitive Radio Design}

Based on the software defined radio (SDR) technology the term cognitive radio (CR) has been developed during the last years. The general approach presented by Mitola [1] describes a high flexible, adaptable and self-learning mobile terminal, which builds a potential precondition for future wireless systems. Besides a number of approaches discussed in the literature regarding the learning and adaptation of CRs to the user's behavior also the integration of dynamic spectrum allocation mechanisms in CRs is an important issue. Due to the high flexibility of the underlying SDR-architecture a dynamic adaptation to the different scenarios, their requirements and channel conditions can be achieved.

As described in section III and discussed in more detail section IV each observed environment is characterized by a different channel occupation density. Especially the scenario of a mega-event requests a high spectral agility in order to support an adaptation also in such a highly varying environment. In this special case a solution could include the allocation of frequencies which are under-utilized in the close vicinity to users geographically located at the edge of the hot-spot. In the near neighborhood of the event there are enough unoccupied frequencies left which could handle the traffic in these areas. 
Of course, this would require the availability of positioning information which additionally increases the effort of CR mobile terminals and CR fixed stations. But a better QoS handling within the hot-spot area due to distribution of the high traffic density to a wider frequency range would be the advantage.

Furthermore, a high agility in the frequency and also in the time domain would be required for the overlay system's terminals. As it could be seen in section IV-D also in a normal urban environment with ordinary traffic volume the average channel occupation of single traffic channels varies between 10 and 60 percent. In order to increase the spectral utilization by using overlay systems without unacceptable interference to the primary system the CR terminals have to have the same order of time resolution as the primary system. Additionally, in order to handle hot-spot scenarios also a wide frequency range has to be monitored in order to be able to adapt the overlay system to the remaining unoccupied frequencies. Moreover, it could be seen that the current environment has to be considered for the definition of the detection threshold within an overlay CR system.

Another property of CRs is the ability for evaluation of situations and drawing conclusions from the situations. In order to realize this kind of machine learning capability several approaches have been presented [12]. Nevertheless, due to the restricted processing capacity within a mobile terminal the mass of informations resulting from the scene analysis needs to be reduced as much as possible. Therefore, the presented classification of different environments and their general characterization using the presented parameters seems to be a first applicable approach.

\section{CONCLUSiON}

Due to the ever increasing demand for wireless communications, efficient use of the spectrum will become mandatory for future wireless techniques. This requires a paradigm shift from the current fixed frequency allocation to an allocation scheme which varies spatially and temporally, thereby accounting for varying demands. Dynamic allocation may, for example, be performed by using spectrum pooling. Thus, powerful methods for the detection and the exploitation of spectrum holes, based on observation of the radio's environment, are compulsory for an efficient use of wireless resources.

The design of powerful algorithms for a more efficient use of radio frequencies has to be based on an extensive analysis of the current situation. Therefore, in this paper measurements are described which determine the power per timefrequency slot in a predefined frequency range. The results of the measurements are analyzed be means of statistics. In order to obtain representative results, different scenarios were considered, describing the mobile communication system for a hot spot scenario, for a cell in close vicinity of a hot spot and for a cell in everyday's life.

In a first step, the distribution of the power per timefrequency slot observed by the measurements is calculated. As a result, the different channels (noise, traffic, beacon) of a mobile communication system could be identified for each scenario. The statistical distribution of the power is described by the cumulative distribution function, the complementary cumulative distribution function and the averaged power histogram, respectively.

In a second step, a detection threshold is calculated which is used for the classification of channels. All time-frequency slots exceeding this threshold are classified as occupied slots, whereas all time-frequency slots below this threshold are classified as unoccupied. The analysis of occupied slots of a channel results in an averaged channel occupation, which may be used for classifying whether or not a channel is suited for dynamic spectrum usage.

Finally, it can be concluded that the observations confirmed the assumption of an artificially created spectrum scarcity. Further analysis of the spectral environment may pave the way to algorithms for a more efficient spectrum usage. Possible approaches may be based on extensive analysis of the run length of occupied and non-occupied slots in order to assess channels, or the mathematical modeling of the observed behavior.

\section{ACKNOWLEDGMENT}

The authors would like to thank Bernd Gutheil from the Technische Universität Kaiserslautern, James Gross from the Technische Universität Berlin for their kind support, and Johannes Fink for his help in preparing the measurements.

\section{REFERENCES}

[1] J. Mitola, "Cognitive radio - An integrated agent architecture for software defined radio," Ph.D. dissertation, Royal Institut of Technology (KTH), Kista, Sweden, 2000

[2] T.A. Weiss, F. Jondral, "Spectrum pooling: an innovative strategy for the enhancement of spectrum efficiency," Communications Magazine, IEEE, vol. 42 , no. 3, pp. 8-14, March 2004.

[3] S. Haykin, "Cognitive radio: brain-empowered wireless communications," Selected Areas in Communications, IEEE Journal on, vol. 23, no. 2, pp. $201-220$, Feb. 2005.

[4] T. Kamakaris, M. M. Buddhikot, and R. Iyer, "A case for coordinated dynamic spectrum access in cellular networks," Proc. IEEE Dynamic Spectrum Access Networks and Technologies (DySPAN '05), pp. 289$298,2005$.

[5] J. O. Juntunen, V. Ranki, and K. Kalliola, "Spectrum measurements and spectrum occupancy," Wireless World Research Forum (WWRF15), Paris, 2005.

[6] M. P. Olivieri, G. Barnett, A. Lackpour, A. Davis, and P. Ngo, "A scalable dynamic spectrum allocation system with interference mitigation for teams of spectrally agile software defined radios," Proc. IEEE Dynamic Spectrum Access Networks and Technologies (DySPAN '05), pp. 170$179,2005$.

[7] T. M. Schäfer, Experimentelle und simulative Analyse der Funkwellenausbreitung in Kliniken. Universität Karlsruhe,Dissertation, 16.06.2003.

[8] C. Rauscher, Grundlagen der Spektrumsanalyse. Rohde \& Schwarz, 2004.

[9] Rohde \& Schwarz, User Manual. Spectrum Analyzer FSL6, 2005.

[10] FCC, "Spectrum policy task force report, ET Docket No. 02-155," Tech. Rep., Nov. 2002.

[11] Shared Spectrum Company, "Comprehensive spectrum occupancy measurements over six different locations," www.sharedspectrum.com, August 2005 .

[12] T. Mitchell, Machine Learning. McGraw-Hill International Editions, 1997. 